\title{
WORLD FEDERATION OF SOCIETIES OF ANAESTHESIOLOGISTS
}

Dr. Harold Griffith reports that plans for the World Federation of Societies of Anaesthesiologists are proceeding. He will attend a meeting of the Organizing Committee in Holland in June, at which reports will be received from national societies in the various countries and plans prepared for the Constituent Assembly of the new Federation. Up to the present, societies of anaesthesiologists in thirty different countries have approved the formation of the Federation and have made application for membership. It appears, therefore, that this will become a really representative organization of all the anaesthesiologists throughout the world.

It should be some satisfaction to Canadians to realize that their society is the third largest national group, and also that they have had an active share in the development of the World Federation. The purpose of the Federation as outlined in the Constitution is:

"To provide better anaesthesia for more people throughout the world."

It is hoped that this purpose may be achieved by setting up standards of training for anaesthesiologists; exchange of information regarding opportunities for training; assistance in the development of national societies in countries where anaesthesiology is in a very primitive state; and advising international and national government bodies on problems relating to anaesthesia.

The new Federation will be controlled entirely by anaesthesiologists, and will not have any official connection with governments, or the World Health Organization, or UNESCO.

The Constituent Assembly of the World Federation of Societies of Anaesthesiologists will be held at Scheveningen, near The Hague, September 5-10, 1955. The various national societies will send official delegates to this assembly. There will be held at the same time and the same place the first World Congress of Anaesthesiologists sponsored by the new Federation. The programme for this World Congress is now being prepared with the Netherlands Society of Anaesthesiologists as hosts. There will be a comprehensive scientific programme, and much opportunity for pleasant social diversion. It is hoped that there will be large delegations present from Canada, the United States and the other Englishspeaking countries. English will be the official language of the Congress, as well as the World Federation. Mr. W. A. Fentener van Vlissingen, Nederlandes Anaesthesisten Vereniging, Butroven, Holland, has been appointed Executive Secretary of the World Congress of Anaesthesiologists and he will be pleased to send information regarding plans for the Congress to anyone who is interested. 


\section{FEDERATION MONDIALE DES SOCIETES D'ANESTHESISTES}

LE Dr. Harold Griffith rapporte que le projet d'établissement d'une Fédération Mondiale des Sociétés d'Anesthésistes se poursuit. Il assistera à une réunion du comité d'organisation en Hollande au mois de juin, réunion à laquelle des rapports seront reçus des sociétés nationales des divers pays et des plans seront établis pour l'Assemblée Constituante de la nouvelle fédération. Jusqu'à présent les sociétés d'anesthésistes de trente pays différents ont approuvé la création d'une fédération et ont demandé à être admises comme membre. Il semble donc que cette Fédération deviendra une organisation vraiment représentative de tous les anesthésistes à travers le monde.

Les Canadiens seront heureux de constater que leur société est par ordre d'importance numérique le troisième des groupes nationaux et aussi qu'ils ont eu une part active au développement de la Fédération Mondiale. Le but de cette Fédération est établi dans la consttution comme suit:

"Fournir une meilleure anesthésie pour un plus grand nombre à travers le monde."

On espère que ce but sera atteint par l'établissement de normes pour la formation d'anesthésistes; par l'échange de renseignements concernant les occasions d'entraînement; par l'assistance au développement de sociétés nationales dans ces pays où l'anesthésie est dans un état encore très primitif; et en conseillant les organismes gouvernementaux, internationaux et nationaux sur les problèmes relatifs à l'anesthésie.

La nouvelle fédération sera contrôlée entièrement par des anesthésistes et n'aura aucun rapport officiel avec les gouvernements ou l'Organisation Mondiale de l'Hygiène ou l'unEsco.

L'Assemblée Constituante de la Fédération Mondiale des Anesthésistes aura lieu à Scheveningen près de La Haye du 5 au 10 septembre, 1955. Les diverses sociétés nationales enverront des délégués officiels à cette assemblée. A la même époque et au même endroit aura lieu le premier Congrès Mondial d'Anesthésistes, une mitiative de la nouvelle fédération. Le programme pour ce Congrès Mondial est maintenant en voie de préparation, la Société d'Anesthésistes de Hollande agissant en capacité d'hôte. Il y aura un programme scientifique complet et beaucoup d'occasions de divertissements agréables. On s'attend à ce qu'il y ait de grandes délégations du Canada, des Etats-Unis et des autres pays de langue anglaise. L'anglais sera la langue officielle du Congrès et de la Fédération Mondiale. M. W. A. Fentener van Vlissingen, Nederlandes Anaesthesisten Vereniging, BщтHоJEN, Hollande, a été nommé secrétaire-exécutif du Congrès Mondial et il sera heureux de transmettre des renseignements au sujet des plans pour le Congrès à tous ceux qui s'y intéressent. 